7. Child Lang. 40 (2013), 307-335. (C) Cambridge University Press 2012. The online version of this article is published within an Open Access environment subject to the conditions of the Creative Commons Attribution-NonCommercial-ShareAlike licence $<$ http://creativecommons. org/licenses/by-nc-sa/2.5/ $>$. The written permission of Cambridge University Press must be obtained for commercial re-use.

doi:10.1017/So30500091 1000444

\title{
Functional reorganization in the developing lexicon: separable and changing influences of lexical and phonological variables on children's fast-mapping*
}

\author{
CRISTINA MCKEAN, CAROLYN LETTS \\ AND DAVID HOWARD \\ Newcastle University
}

(Received 3 October 2010-Revised 20 April 201 1 -Accepted 2 I September 201 IFirst published online 20 fanuary 2012)

\begin{abstract}
Neighbourhood Density (ND) and Phonotactic Probability (PP) influence word learning in children. This influence appears to change over development but the separate developmental trajectories of influence of $\mathrm{PP}$ and ND on word learning have not previously been mapped. This study examined the cross-sectional developmental trajectories of influence of PP and ND on fast-mapping in thirty-eight English-speaking children aged $3 ; \mathrm{OI}-5 ; 02$, in a task varying $\mathrm{PP}$ and $\mathrm{ND}$ orthogonally. PP and ND exerted separable influences on fast-mapping. Overall, low ND supported better fast-mapping. The influence of PP changed across the developmental trajectory, 'switching' from a high to a low PP advantage. A potential explanation for this 'switch' is advanced, suggesting that it represents functional reorganization in the developing lexicon, which emerges from changes in the developing lexicon, as phonological knowledge is abstracted from lexical knowledge, over development.
\end{abstract}

\section{INTRODUCTION}

The apparent ease with which young children learn words belies the complexity of the processes involved. In fact, multiple speech and cognitive processing abilities are called into action to learn a new word and numerous constraints and biases exert influence on the process (Golinkoff et al., 2000). An additional source of complexity is that these constraints, biases and processing abilities change across development, exerting differing effects at

[*] Thanks go to the schools, families and children in the North of England who participated, to the ESRC for funding this research and the larger study of which it is a part and to Professor James Law and Dr Helen Stringer for their helpful comments on earlier drafts of this article. Also many thanks go to the two anonymous reviewers whose comments were invaluable in preparing this article for publication. 
different stages in development. A number of models of the developing lexicon suggest these changes in processing abilities and biases arise from changes in the nature of the child's existing lexical knowledge, therefore, over development, a bi-directional and dynamic relationship of influence between lexical knowledge and speech processing emerges (Garlock, Walley \& Metsala, 200 I Pierrehumbert, 2003; Walley, Metsala \& Garlock, 2003; Werker \& Curtin, 2005). This article seeks to add to our understanding of the nature of such changes in the developing lexicon by exploring change in the influence of lexical and phonological variables on word learning across development.

\section{Models of the developing lexicon}

In a number of models of the developing lexicon, speech processing and, in turn, word learning are influenced by the changing nature of existing lexical knowledge (Garlock et al., 200 I ; Pierrehumbert, 2003; Walley et al., 2003; Werker \& Curtin, 2005). In these models the developing lexicon is thought to differ from the adult lexicon, not simply in terms of size, but also in terms of organizational structure, the degree of specification of lexical representations and the nature of any sublexical or phonemic level representations. Converging evidence from a number of experimental paradigms suggest that, as a child's lexicon grows, the structure and functioning of their speech processing mechanism changes, with developing lexical knowledge seen as the driver of phonological development (Beckman \& Edwards, 2000; Garlock et al., 200 I ; Pierrehumbert, 2003; Walley et al., 2003; Werker \& Curtin, 2005). That is, over development, vocabulary growth and the associated increasing density in similarity neighbourhoods of lexical representations provides the impetus for the emergence of phoneme level representations. Phoneme categories do not emerge 'fully formed'; rather there is a process of emergence from broader categories of sublexical representations to fully specified categories of phonemes. There are a number of possible pathways for the emergence of phoneme categories, with a number of researchers positing a process where sublexical level representations become more fine-grained, moving through syllable, onset + rime, onset + vowel + coda representations until finally specifying to the level of phoneme representations (De Cara \& Goswami, 2002; 2003; Ziegler \& Goswami, 2005); a process often described as 'lexical restructuring' (Walley et al., 2003). The emergence of phoneme representations allows the child to produce and process phonemes independently of the words in which they occur and so become more efficient and flexible in their speech processing (Beckman \& Edwards, 2000; Garlock et al., 200I; Walley et al., 2003; Werker \& Curtin, 2005). The speech processing mechanism can therefore be seen as both the product of and the 
mechanism for word learning. This circularity of interdependence predicts that children at different stages of lexical development will approach word learning with radically different speech processing architectures, and so understanding the nature of these different mechanisms across the developmental trajectory is essential if we are to understand the nature of word learning.

A recent development in this field is the PRIMIR framework (Processing Rich Information from Multi-dimensional Interactive Representations) advanced by Werker \& Curtin (2005), which makes explicit links between speech processing and word learning over development. This framework suggests that during speech processing, multiple levels of representation are available to the child; semantic, phonetic/indexical, word forms and phonemes (the latter emerging over development). Speech is processed using these representations in parallel and interactively. Information is encoded in a distributed way and so behaviour is determined by the global activity of the network, not a single part of it. Hence the process often conceptualized as 'lexical restructuring' is a function of changes in the structure of the WHOLE LEXICON as a result of additional lexical representations being added to the network.

Werker and Curtin (2005) also suggest that an additional layer of complexity exists in children's speech processing. That is, that different task demands act as a filter, differentially directing the child's processing 'attention' to different aspects of the representations available to support speech processing. In support of this argument, Werker and Curtin cite apparently contradictory results regarding infants' abilities to discriminate fine-grained distinctions between words. That is the finding that infants aged $\mathrm{I} ; 2$ who confuse phonetically similar words when they are linked to objects, can discriminate them when they are not (Werker \& Yeung, 2005). Werker and Yeung suggest that this difference arises from differences in the overall processing demands of the tasks. In order to understand the developmental trajectory of speech processing, therefore, consideration must be given to three fundamental areas: the changes occurring at ALL LEVELS of representation; the nature of the INTERACTIVITY between the levels of representation; and the nature of the biases brought to bear on processing as a function of the TASK.

\section{Creating a window into the developing lexicon: lexical and phonological variables}

These models, and the evidence upon which they draw, demonstrate that lexical, phonological and phonetic levels of processing interact in speech processing and word learning and also that the way in which they contribute changes across development. In order to uncover the nature of lexical 
and phonological processing, researchers exploring adult speech processing have manipulated the lexical variable of Neighbourhood Density (ND) and the phonological variable of phonotactic probability (PP). PP is a measure of the likelihood or frequency of particular sequences of sounds in a language (Vitevitch, Luce, Pisoni \& Auer, I999). ND is a measure of the numbers of lexical neighbours in the similarity neighbourhood of a word and is usually calculated as the number of words differing from the target by one phoneme substitution, addition or deletion (Vitevitch et al., I999). PP and ND are highly correlated and in most studies are covaried. In adult processing it is thought that these variables exert influence at different levels of speech processing (Vitevitch \& Luce, I 998; I999). In spoken word recognition, members of the similarity neighbourhood compete with one another, and so words which reside in 'dense' neighbourhoods (those with many neighbours) are recognized more slowly and less accurately than those in more 'sparse' neighbourhoods (Luce \& Pisoni, I998; Vitevitch et al., I 999). At the phonological level of processing, high PP has been shown to speed processing in such tasks as same/different judgements and non-word repetition (Vitevitch \& Luce, I 999; 2005). However, in other tasks, such as lexical decision tasks and real word repetition, higher PP is associated with slower processing (Vitevitch \& Luce, I 999; Vitevitch et al., I 999). This result has been explained due to the high correlation between ND and PP. That is, tasks such as lexical decision and real-word repetition bias processing toward the lexical level such that the inhibitory effects of high ND predominate and tasks such as same/different judgements and non-word repetition bias processing toward the phonological level such that PP effects predominate (Vitevitch \& Luce, I 999; Vitevitch et al., I 999).

Exploring the separate influence of ND and PP on word learning in children, and the nature of change in their influence across development, could allow us to explore a number of predictions made by the PRIMIR framework of speech processing. The framework predicts that, during word learning, multiple levels of representation affect the word learning process (semantic, phonetic/indexical, word forms and sublexical representations), that these different levels of representation interact with one another, that the nature of the representations and the nature of the interactions change across development, and, potentially, different aspects of the process of learning a word may focus 'attention' on different aspects of the representations available to support speech processing. Based on the assumption that PP and ND tap differing levels of representation, then the PRIMIR framework would predict that the influence of PP and ND on word learning will BE SEPARABLE, INTERACTIVE, WILL CHANGE WITH AGE, AND WILL BE AFFECTED BY THE NATURE OF THE TASK.

The framework does not make detailed predictions regarding how PP and ND affect word learning; however inferences can be drawn from the model 
as to the likely pattern of change in the influence ND over development. Werker and Curtin (2005) see the challenge of word learning as having a number of components, including: (I) segmenting the speech stream; (2) creating a lexical representation of the phonetic detail of the word form; (3) creating a conceptual representation of the referent; (4) linking the word form to the referent; and (5) holding this link in memory to result in a meaningful word. This is a computationally highly demanding process which is differentially influenced by the ND of the 'to be learned' word depending on whether phoneme representations have emerged. That is, in infants, where phoneme categories are not present, high ND will inhibit learning as the child does not know which aspects of the lexical representation which they are creating are 'criterial' in terms of making it meaningfully distinct from other words which they know or are in their environment (e.g. voice onset time rather than pitch). They therefore will not direct their limited processing resources to this 'criterial' information but rather to multiple aspects of the word form which may or may not be relevant. This more diffuse deployment of attentional resources therefore leaves fewer resources available to create form-referent mappings and commit them to memory. Where words are highly distinct from one another or from known words (e.g. duck, ball), then mapping these to distinct referents is a relatively trivial task. However, where there is a significant degree of overlap (e.g. pin versus bin), then the child must process highly detailed phonetic information to determine where the differences lie, leaving inadequate attentional resource available to complete the form-referent mapping required to learn the word.

Once the child begins to develop phoneme categories, these representations focus the child's attention onto JUST that information which is relevant for the word-referent mapping to occur, so leaving sufficient computational resources to learn the word. Therefore, at this later developmental stage, high ND should cease to be a disadvantage, and could, in fact, become an advantage if, as a number of models of speech processing posit (Beckman \& Edwards, 2000, Pierrehumbert, 2003), it predicts more robustly defined phoneme representations with which to focus attentional resources.

Mapping the developmental trajectory of influence of ND and PP on word learning could allow us to explore if and how differing levels of representation interact with one another during word learning, and if and how the nature of those representations and their interactions change across development.

\section{The influence of ND and PP on word learning in children}

It is still unclear, from the current available evidence, whether ND and $\mathrm{PP}$ exert separable influences on word learning in children, whether these 
influences change across development and whether they differ for different stages of the word learning process (e.g. fast mapping: the first stages of word learning occurring in the first few exposures to a novel word, vs. slow mapping: the process of elaborating and adding detail to an existing representation over a number of exposures). One major reason for the absence of a clear picture is the high degree of correlation between ND and PP. The majority of experimental studies have covaried these factors, making it impossible to be sure whether it is PP, ND or an interaction between the two variables which is responsible for the effects observed (Storkel, 2001; 2003; Storkel \& Rogers, 2000).

Second, the range of methodologies applied to this question makes comparisons between studies difficult, especially as differing task demands probably bias an individual's processing 'attention' towards different representations (Vitevitch \& Luce, I999; Werker \& Curtin, 2005). Therefore, small differences in either the task presentation or the dependent variable used as a measure of word learning could affect whether or not PP and ND are shown to exert an influence. For example, fast-mapping studies in children between the ages of three and thirteen years have shown that this age group of children learn words with high PP/high ND more easily than those with low PP/low ND (Storkel, 200I; 2003; Storkel \& Rogers, 2000). In contrast, studies with infants (aged $\left.I_{5} ; 5^{-I} ; 6\right)$, which use picture fixation or preferential looking paradigms, have demonstrated both a low ND advantage (Swingley \& Aslin, 2006) and a high PP/low ND advantage (Hollich, Jusczyk \& Luce, 2002). Furthermore, a study of adults' fastmapping, which used an expression probe as the measure of learning, found a low PP/high ND advantage (Storkel, Armbruster \& Hogan, 2006). Whether these differences in results represent changes in the influence of ND and PP across the developmental trajectory, from infant to adult, or whether they simply reflect differences in experimental methodologies, remains unclear.

In order to address the issue of developmental change in word learning biases, researchers have also examined the nature of change in children's vocabulary knowledge, using the characteristics of the words in children's productive lexicons to uncover changes in the biases that children bring to bear on the word learning process. Maekawa and Storkel (2006) examined the productive vocabularies of individual children, using analysis of language samples from the CHILDES database (MacWhinney, 2000) and Storkel $(2009 ; 2004)$ considered the issue with respect to large cohorts, using parentreported data from the CDI (Dale \& Fenson, I996; Fenson et al., I997).

Findings from individual case studies of children aged I ; 4, I; 9 and I ; I over a one-year period (Maekawa \& Storkel, 2006) suggest there are large individual differences and changes over time in the influence of PP and ND, with high and low ND helping word acquisition at different stages of the 
trajectory and high PP facilitating word learning for the youngest child studied. Analysis of CDI data, on the other hand, suggest that for children aged between I; 0 and 2;6 (Storkel, 2009; 2004) low PP and high ND facilitate word learning.

From this data it is therefore extremely difficult to create a hypothetical developmental trajectory of the changing influences of ND and PP on word learning in children. As Storkel states: 'ultimately, additional data are needed from studies that systematically vary these factors while examining learning of words fully crossed in phonotactic probability and neighbourhood density to more clearly determine when and how each variable influences word learning by infants' (2009: 3 I4).

\section{The aims of the current study}

This study answers Storkel's call for direct experimental evidence through examining the fast-mapping abilities of typically developing children with stimuli that vary PP and ND orthogonally. In addition, to explain the data fully, changes in their influence across development must also be considered to determine whether PP and ND have a stable or a changing trajectory of influence on the process of fast-mapping. This in turn can provide a window on the changing nature of lexical and sublexical representations used to support speech processing and hence word learning.

Studying longitudinal change is, however, challenging. Prospective longitudinal cohort studies are obviously the gold standard for such research However, such methodologies have difficulties: they are highly resource intensive, vulnerable to subject drop-out and yield results slowly. In order to invest in prospective longitudinal data collection, the value of the methodologies, measures and research questions, and the case for a longitudinal perspective, need first to be empirically demonstrated.

This study used a cross-sectional methodology to investigate developmental change in fast-mapping biases and to gather empirical data to support the case for future longitudinal study. One approach to analyzing such data is Thomas's cross-sectional trajectory analysis (Thomas, Annaz, Ansari, Scerif, Jarrold \& Karmiloff-Smith, 2009). Thomas advocates the use of linear regression and ANCOVA to create developmental trajectories for groups of children from cross-sectional data to test predictions about longitudinal change. We applied this approach to our data in order to answer the following research questions:

I. Do phonological (PP) and lexical (ND) variables exert separable influences on children's fast-mapping abilities?

2. Do these influences change across development?

3. What do these changes tell us about the structure and functioning of the developing lexicon in typical development? 
TABLE I. Numbers of participants by age group

\begin{tabular}{llcl}
\hline $\begin{array}{l}\text { Age range } \\
\text { (months) }\end{array}$ & $\mathrm{N}$ & $\begin{array}{c}\text { Age range } \\
\text { (months) }\end{array}$ & $\mathrm{N}$ \\
\hline $37-40$ & 3 & $52-55$ & 6 \\
$4 \mathrm{I}-44$ & 2 & $56-59$ & 8 \\
$45-47$ & 7 & $60-62$ & 6 \\
$48-5 \mathrm{I}$ & 6 & & \\
\hline
\end{tabular}

TABLE 2. Participant summary characteristics

\begin{tabular}{|c|c|c|c|c|}
\hline & $\begin{array}{c}\text { Age } \\
\text { (months) }\end{array}$ & $\begin{array}{c}\text { RDLS III } \\
\text { Comprehension Scale } \\
\text { centile scores }\end{array}$ & $\begin{array}{c}\text { RDLS III } \\
\text { Expression Scale } \\
\text { centile scores }\end{array}$ & $\begin{array}{c}\text { BAS } \\
\text { Block Building Subtest } \\
\text { centile scores }\end{array}$ \\
\hline $\begin{array}{l}M \\
S D\end{array}$ & $\begin{array}{l}5 \mathrm{I} \cdot 68 \\
(7 \cdot 33)\end{array}$ & $\begin{array}{c}65 \cdot 92 \\
(20 \cdot 50)\end{array}$ & $\begin{array}{c}46 \cdot 32 \\
(2 \mathrm{I} \cdot 09)\end{array}$ & $\begin{array}{c}44 \cdot 5 \\
(26 \cdot 22)\end{array}$ \\
\hline
\end{tabular}

NOTE: RDLS III=Reynell Developmental Language Scales III (Edwards et al., I997); $\mathrm{BAS}=$ British Ability Scales (Elliot et al., I 996).

\section{METHOD}

\section{Participants}

The participants were thirty-eight monolingual English speakers in the age range 3;OI-5;O2 with no identified developmental or language difficulties. The number of participants in each age group is presented in Table I.

The children's language, non-verbal skills and hearing status were assessed using the Reynell Developmental Language Scales III (RDLS III : Edwards, Fletcher, Garman, Hughes, Letts \& Sinka, I997), the British Ability Scales Block Building Subtest (BAS: Elliot, Smith \& McCulloch, I996) and the ASHA Audiologic Screening Protocol (ASHA, I997), respectively. Summary data is presented in Table 2.

\section{General procedures}

Ethical approval for this work was obtained and written consent gained from the parents/carers of the children. The children were seen a quiet area in their school for a series of short sessions. The length of the session was tailored to the child's individual level of attention and motivation.

\section{The word learning task}

The fast-mapping task presented in Storkel (200I) was adapted to include stimuli orthogonally varying $\mathrm{ND}$ and $\mathrm{PP}$ and simplified to minimize the 
effect of language knowledge on performance across the trajectory (see 'Appendix').

The children were introduced to the non-word-referent pairings in a story involving two aliens going shopping. At each new location the aliens each bought or used a new referent from one of the categories; buying a toy each at the toy shop, a pet each at the pet shop, eating some food at the café and catching a rocket home at the 'rocket stop'.

During the story each word-referent pairing was presented eight times. To increase the number of repetitions while maintaining the child's attention, a 'storyboard activity' was also completed. This provided the opportunity for two additional repetitions of the word-referent pairings. The storyboard activity involved the children re-enacting the story by moving small figures of Jim and Bob along a board with a street depicted on it, containing each of the locations from the story (the toy shop, the pet shop, the café, the 'rocket stop'). The children were encouraged to move Jim and Bob along the street, to pause at each location, to find the objects which Jim and Bob had bought at that location in the story, and to give the correct object to the correct alien. At each location, the children were presented with a choice of objects including both the eight objects from the story and an additional eight 'alien' objects which did not appear in the story, and at this point heard the appropriate novel words repeated an additional two times. The story and storyboard script can be found in the 'Appendix'. The protocol was administered 'live voice' as, during piloting, this was found to be the most successful method for maintaining the attention of the youngest children through the whole experimental protocol.

The story structure and sentence structures were designed to be as simple as possible. The novel word was presented in sentence-final position and the carrier sentences contained a maximum of three clause elements in order to minimize the possible confound of level of grammatical knowledge on the word learning process across the developmental trajectory (e.g. 'Jim likes the teIn' 'Here's the horf').

\section{The non-words}

A range of $\mathrm{CVC}$ non-words were created using only early developing consonants ( $\mathrm{m} \mathrm{n} \mathrm{p} \mathrm{b} \mathrm{t} \mathrm{d} \mathrm{kg} \mathrm{s} \mathrm{f} \mathrm{w} \mathrm{j} \mathrm{and} \mathrm{h)} \mathrm{(2;o6-3;o6)} \mathrm{(Grunwell,} \mathrm{I} \mathrm{985)} \mathrm{in}$ order to minimize the influence of any phonological processes which may have affected the ability of the youngest children to produce the words. The Phonotactic Probability and Neighbourhood Density of the candidate nonwords were calculated and stimuli chosen varying PP and ND orthogonally with two exemplars of each of the four subcategories in the story (toys, pets, food, vehicles). 
Phonotactic Probability. The PP of the non-words was calculated from data in the CELEX database (Baayen, Piepenbrock \& Gulikers, I995) using the following approach.

For a syllable $\mathrm{C}_{1} \mathrm{~V}_{1} \mathrm{C}_{2}$, the $\mathrm{PP}=\mathrm{p}_{1} \mathrm{p}_{2} \mathrm{p}_{3} \mathrm{p}_{4}$, where:

$\mathrm{p}_{1}=$ probability of $\mathrm{C}_{1}$ given that it follows a syllable (or word) boundary $=$ summed frequency of syllables beginning with $\mathrm{C}_{1} /$ summed frequency of syllables in the database;

$\mathrm{p}_{2}=$ probability of $\mathrm{V}_{1}$ given that it follows a $\mathrm{C}_{1}$ in a syllable onset $=$ summed frequency of syllables with the sequence $\mathrm{C}_{1} \mathrm{~V}_{1} /$ summed frequency of syllables with $\mathrm{C}_{1}$ in the onset;

$\mathrm{p}_{3}=$ probability of $\mathrm{C}_{2}$ given that it follows $\mathrm{V}_{1}$ as the syllable nucleus $=$ summed frequency of syllables with the sequence $\mathrm{V}_{1} \mathrm{C}_{2}$ /summed frequency of syllables with $\mathrm{V}_{1}$ as nucleus;

$\mathrm{p}_{4}=$ probability of a syllable (or word) boundary given that it follows a $\mathrm{C}_{2}$ in a syllable coda $=$ summed frequency of syllables with the sequence $\mathrm{C}_{2}$-/ summed frequency of syllables with $\mathrm{C}_{2}$ in the coda (where-indicates a syllable (or word) boundary).

This method considers the probability of sequences of phonemes, taking into account where the phonemes occur relative to the syllable nucleus. The CELEX calculations were triangulated using Vitevitch's online 'Phonotactic Probability Calculator' (Vitevitch \& Luce, 2004) (see Figure I).

Neighbourhood Density. Neighbourhood Density was defined as the number of neighbours differing from the target by one phoneme substitution, addition or deletion and was also calculated using the CELEX database (Baayen et al., r995), considering only the words in the database with a frequency of greater than I per i $6 \cdot 9$ million words.

An estimate of high and low neighbourhood density for young children was developed determined by considering the characteristics of the threephoneme words in the Morrison, Chappell and Ellis (1997) word list, with an age of acquisition of less than five years, and the three-phoneme nouns in the British English version of the CDI (Fenson et al., I 997). The mean and standard deviation of the ND for these words was calculated $(\mu=23.7$; $S D=8$ ). As a result we operationally defined High ND as $\geqslant$ the mean and Low ND $\leqslant$ I SD below the mean. These criteria ensured that the chosen stimuli were substantially different from one another in ND whilst also producing sufficient numbers of candidate stimuli.

Stimulus selection. For the high ND /high PP and low ND/low PP nonwords, the stimuli with the highest and lowest possible values respectively for each measure were chosen. For the low ND/high PP and high ND/low PP stimuli, those non-words which fulfilled the ND criteria for high and low status described above were identified and then, from those stimuli, those with the lowest or highest possible PP values chosen. 


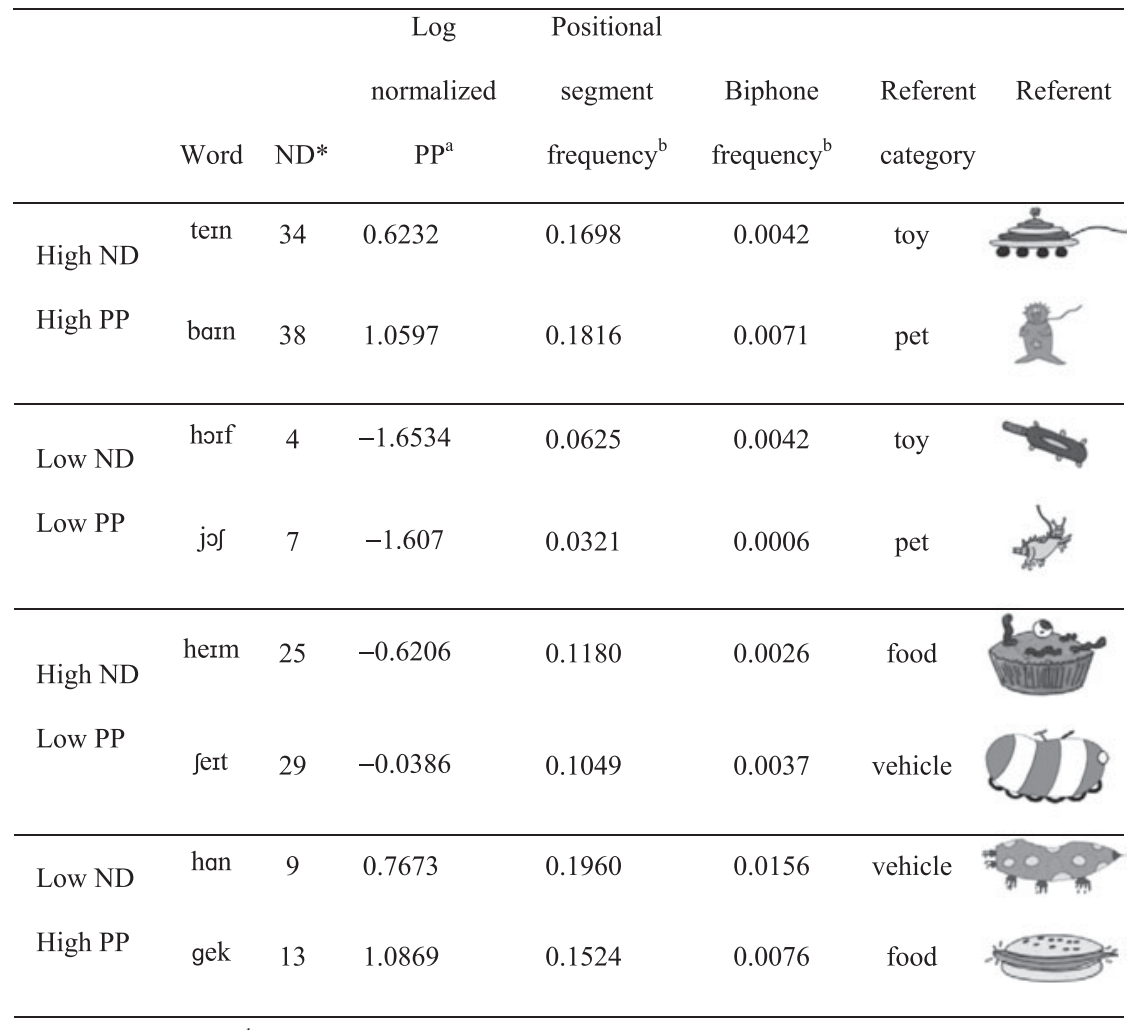

NOTES: ${ }^{\mathrm{a}}$ CELEX; ${ }^{\mathrm{b}}$ Hoosier Mental Lexicon.

Fig. I. Form and referent characteristics of the chosen stimuli.

Stimuli were chosen to maximize the number of different phonemes used across the non-words in order to make the words maximally distinct. It was not possible, however, to create stimuli with no overlap in the phonemes used due to the additional stimulus constraints. The final stimuli, therefore, were chosen to contain the fewest overlapping phonemes whilst containing only early developing phonemes and varying orthogonally PP and ND.

Two-sample $t$-tests demonstrated that the differences between the stimuli categorized as 'high' and 'low' PP and ND were statistically significant (ND: $t(6)=6 \cdot 8 \mathrm{I}, p<0 \cdot 00 \mathrm{I}$ (one-tailed); PP log normalized: $t(3 \cdot 49)=4.55$ $p=0.007$ (one-tailed); PP positional segment frequency: $t(6)=4.39$, $p=0.002$, (one-tailed); PP biphone frequency: $t(6)=2 \cdot 28, p=0.032$ (onetailed)). 
The referents. The eight resulting novel non-words were each paired with a novel referent in a story context. The story was set on an alien planet and the novel objects represented items from the categories 'alien' toys, food, pets and vehicles with two items in each semantic category (see Figure I). The semantic categories were chosen from those which appear in the CDI and so exist in the lexicon of typically developing children aged 2; o6 (Fenson et al., I 997). Novel words were assigned to the referents so that for each semantic category pair (toys, foods, pets, vehicles) the novel word pair contrasted high and low PP and high and low ND (see Figure I). To ensure that there were no significant differences in PP and ND characteristics between the categories, a series of one-way ANOVAs were completed. There was no main effect of semantic category for any of the ND or PP metrics (ND: $F(3,4)=0.02, p=0.995$, partial $\eta^{2}=0.02 ;$ PP $\log$ normalized: $F(3,4)=0.17, p=0.9 \mathrm{I}$, partial $\eta^{2}=0.12$; PP positional segment frequency: $F(3,4)=0.14, p=0.93$, partial $\eta^{2}=0.10$; PP biphone frequency: $F(3,4)=0.55, p=0.67$, partial $\left.\eta^{2}=0.29\right)$.

The semantic categories and referents were not counterbalanced across participants. Tests exploring the potential confounds of items and semantic categories were completed post hoc and are presented with the results.

\section{The measures of learning}

The children's knowledge of the newly learned words was assessed using a comprehension and expression probe; the comprehension probe consisting of the child finding the correct referent from a choice of four pictures ('show me the $\mathrm{X}$ ') and the expression probe being an invitation to name a set of ten object pictures which included the newly learned referents, together with eight additional early developing nouns. The probes were presented at three points in the experiment: for each new category pair after the story episode which introduced them (i.e. the toys were tested after the toy shop visit, the pets after the pet shop visit, and so on); for all eight novel words at the end of the story; and again for all eight words at the end of the storyboard game. The expression probe was presented first at each testing point so that the additional repetitions of the words during the comprehension probe did not influence the children's responses. The children were not given feedback regarding the accuracy of their responses, but were praised for their effort and concentration. The children were extremely reluctant to respond to the expression probe, performing close to floor in most cases and so further results will not be presented here (McKean, 2009). The overall structure of the task is outlined in Figure 2.

The comprehension probe. The comprehension probe tested the abilities of the children to fast-map a representation of the phonological string for the novel word (a lexical representation), a representation of the referent for the 


\begin{tabular}{|c|c|c|}
\hline Context & Exposures & $\begin{array}{l}\text { Expression and } \\
\text { comprehension }\end{array}$ \\
\hline \multirow{14}{*}{ Story } & $\begin{array}{l}2 \times \text { toys } \\
3 \text { repetitions }\end{array}$ & probe \\
\hline & & 1. Toys \\
\hline & $\begin{array}{l}2 \times \text { toys } \\
3 \text { repetitions }\end{array}$ & \\
\hline & $\begin{array}{l}2 \times \text { pets } \\
3 \text { repetitions }\end{array}$ & \\
\hline & & 2. Pets \\
\hline & $\begin{array}{l}2 \times \text { pets } \\
3 \text { repetitions }\end{array}$ & \\
\hline & $\begin{array}{l}2 \times \text { food } \\
3 \text { repetitions }\end{array}$ & \\
\hline & & 3. Food \\
\hline & $\begin{array}{l}2 \times \text { food } \\
3 \text { repetitions }\end{array}$ & \\
\hline & $\begin{array}{l}2 \times \text { vehicles } \\
3 \text { repetitions }\end{array}$ & \\
\hline & & 4. Vehicle \\
\hline & $\begin{array}{l}2 \times \text { vehicles } \\
3 \text { repetitions }\end{array}$ & \\
\hline & $\begin{array}{l}\text { All novel words } \\
2 \text { repetitions }\end{array}$ & \\
\hline & & 5. All novel words \\
\hline \multirow{2}{*}{$\begin{array}{r}\text { Story } \\
\text { board } \\
\text { game }\end{array}$} & $\begin{array}{l}\text { All novel words } \\
2 \text { repetitions }\end{array}$ & \\
\hline & & 6. All novel words \\
\hline
\end{tabular}

Fig. 2. Schedule of exposures and word learning measures in the fast-mapping task.

novel word (a semantic representation), and a link between the two representations. The child was asked to 'show me the $\mathrm{X}$ ' and selected the corresponding picture from a choice of four: (I) the Target referent; (2) a Related Distracter (the referent from the same semantic category in the story); (3) an Unrelated Distracter (another referent from the story from a different semantic group); and (4) a Foil (a novel object which did not appear in the story).

Choosing the Targets at a level above chance would suggests the child had successfully created both a lexical and a semantic representation, which were sufficient for him/her to recognize the word on a subsequent presentation, and link it to its correct referent. Choosing the Related Distracter at a level above chance would suggest the child had again created a lexical representation which was sufficient for recognition but had either 
created a connection to the incorrect semantic representation (the other object in the semantic category), or to the semantic category without being sure of the specific referent. The child had therefore made a link between the lexical representation and the sEMANTIC CATEGORY of the two novel referents but not to the specific semantic representation.

Choosing the Unrelated Distracter would most likely represent a child who was guessing. However, to make this response at a level above chance could suggest that the child had created a lexical representation sufficient to recognize it again, but that this representation was not linked to a specific semantic representation. Rather, it was linked to the semantic category 'appears in the story'; a network created between the newly learned referents.

Choosing the Foil would also most likely suggest that the child was guessing. However, if this choice occurred at a level above chance, it may suggest that the child had not created a lexical representation of the word at all and so responded as though they have never heard the word before, assuming that this 'novel word' must refer to a novel referent, so choosing the Foil.

Scoring. Responses on the comprehension probe were recorded by circling the child's response on a score sheet. Reliability of coding was not measured as it was thought to be unnecessary for coding of a pointing response. Totals for each response (Target, Related Distracter, Unrelated Distracter and Foil) were tallied across the three assessment points. There was no significant difference in scores at each time point and so total number of responses for each response type was used for all analyses (see 'Appendix', Table 3).

\section{Data analysis}

The analytical approach chosen was Thomas's 'Trajectory Analysis' (Thomas et al., 2009). This method applies linear regression and ANCOVA to create developmental trajectories for groups of children from crosssectional data. To that end a series of regression analyses and within-subject ANOVAs and ANCOVAs with age as the covariate were completed in order to consider the influence of development and phonological (PP) and lexical (ND) variables on the fast-mapping abilities of the children.

\section{RESULTS}

The influence of PP, ND and of developmental change on the fast-mapping biases of the children are described below; first with reference to overall fast-mapping ability, as measured by the number of correct responses (Target) in the comprehension probe, and second with reference to their 


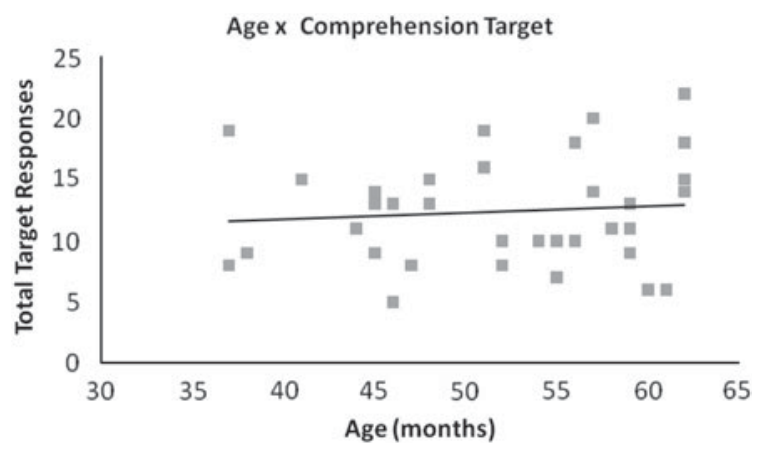

Fig. 3. Relationship between comprehension probe scores (choosing the Target) and age.

influence on the semantic networks being created by the children as measured by the errors on the comprehension probe.

\section{Overall fast-mapping ability}

Linear regression analysis showed no significant relationship between age and accuracy of choosing the Target in the comprehension probe $\left(R^{2}=0.0 \mathrm{I}\right.$, $F(\mathrm{r}, 37)=0.3 \mathrm{I}, p=0.58$ ) (see Figure 3 ). The children were therefore not improving in their overall ability to fast-map novel words over the age range studied $(3 ; \mathrm{I}-5 ; 2)$.

The influence of PP on the abilities of the children to identify correctly the target in the comprehension probe across development was analyzed using a one-way within-subject ANCOVA with PP as the within-subjects factor and age as the covariate. Phonotactic probability exerted a significant influence on the children's ability to fast-map the novel words correctly such that low PP words had an overall advantage $(F(\mathrm{r}, 36)=4 \cdot 29, p=0 \cdot 046$, partial $\eta^{2}=0^{\cdot}$ I I $)$. However, there was a significant $\mathrm{PP} \times$ Age interaction; the younger children demonstrating a high PP advantage and the older children demonstrating a low PP advantage $\left(F(\mathrm{I}, 36)=6 \cdot \mathrm{I}_{5}, p=0.02\right.$, partial $\eta^{2}=0 \cdot$ I $_{5}$ ) (see Figure 4).

One-way within-subject ANCOVA analysis with ND as the withinsubjects factor and age as the covariate demonstrated that Neighbourhood Density exerted a significant influence on the children's ability to fast-map the novel words correctly, such that low ND words had an overall advantage $\left(F(1,36)=7.53, p=0.01\right.$, partial $\left.\eta^{2}=0.17\right)$. Inspection of the trajectory of influence of ND suggested that the disadvantage to fastmapping exerted by high ND may be reducing across development (see Figure 5). However, the $\mathrm{ND} \times$ Age interaction was not significant $\left(F\left(\mathrm{r}, 3^{6}\right)=3 \cdot 28, p=0.08\right.$, partial $\left.\eta^{2}=0.08\right)$. 


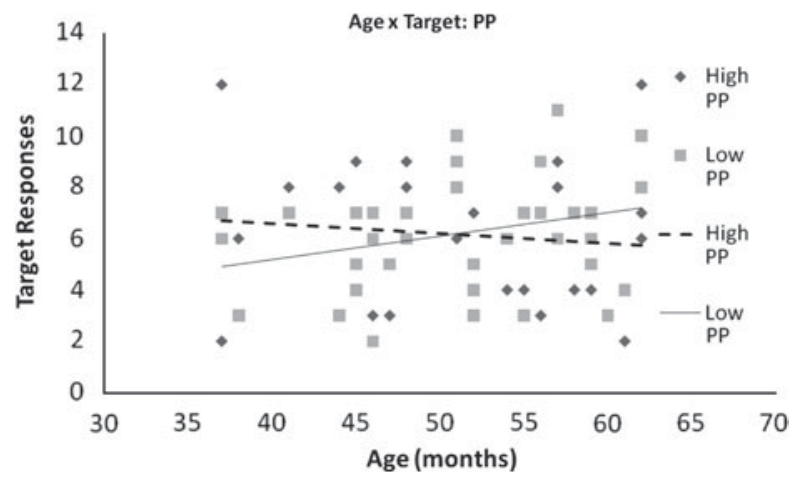

Fig. 4. Trajectory of influence of PP on fast-mapping ability as measured by number of correct (Target) responses on the comprehension probe.

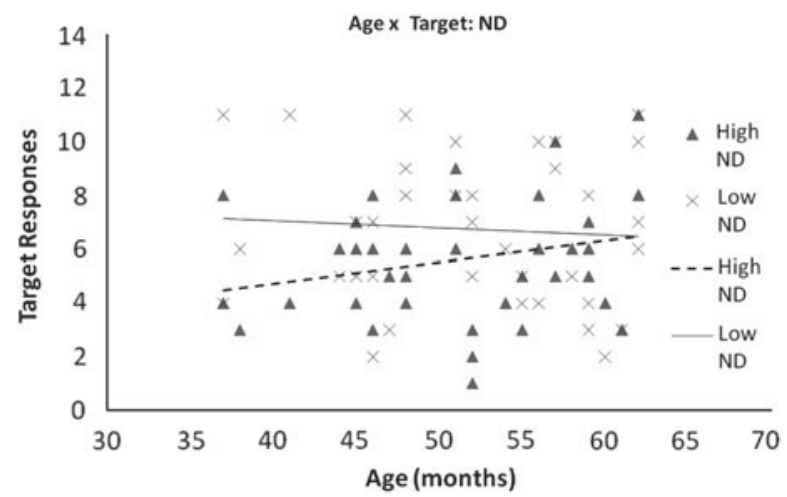

Fig. 5. Trajectory of influence of ND on fast-mapping ability as measured by number of correct (Target) responses on the comprehension probe.

Taken together these results indicate that the children were not improving in their overall ability to fast-map novel words over the age range studied $(3 ; 0 \mathrm{I}-5 ; 02)$. However, changes in the influence of PP show that there are changes in the nature of the processing abilities and biases brought to bear on the fast-mapping process across this age range. In addition, there were separate and distinct effects of ND and PP on the process of fastmapping in children.

\section{Error data}

The developmental trajectory of the influence of PP and ND on error types was considered using a series of one-way ANCOVAs with either PP or ND 
as the within-subject factor and age as the covariate. The trajectory of influence of PP and ND on the frequency of Unrelated Distracter (a referent from the story from a different semantic group from the target) and Foil (a novel object which did not appear in the story) errors was simply a mirror of those for the choosing the Target. Hence for PP, as the children's tendency to learn high PP targets decreased with age the tendency to make high PP Foil and Unrelated Distracter errors increased $\left(\mathrm{PP} \times\right.$ Age $: \mathrm{UD}: F(\mathrm{I}, 36)=\mathrm{I} 0.25, p<0.0 \mathrm{I}$, partial $\eta^{2}=0.22 ; \mathrm{F}: F(\mathrm{I}, 36)=$ $3.80, p=0.059$, partial $\left.\eta^{2}=0.10\right)$, and for ND the overall low ND advantage evinced for choosing the Target was mirrored by increased Unrelated Distracter and Foil errors for high ND words (Main effect ND: UD: $F\left(\mathrm{I}, 3^{6}\right)=4 \cdot 76, p=0.04$, partial $\eta^{2}=0 \cdot \mathrm{I} 2 ; \mathrm{F}: F(\mathrm{I}, 36)=4 \cdot 76, p=0 \cdot 04$, partial $\left.\eta^{2}=0.12\right)$.

The developmental trajectory of influence of PP and ND on the Related Distracter errors (the referent from the same semantic category in the story as the Target) differed from all other response types. For Related Distracter errors there was no significant effect of PP or ND and their influence did not change across the trajectory (PP: $F(\mathrm{~s}, 36)=0.96, p=0.34$, partial $\eta^{2}=0.03$; PP $\times$ Age: $F(\mathrm{I}, 36)=0.79, p=0.38$, partial $\eta^{2}=0.02 ; \mathrm{ND}$ : $F\left(\mathrm{I}, 3^{6}\right)=0.17, \quad p=0.69, \quad$ partial $\eta^{2}=0.0 \mathrm{I} ; \quad \mathrm{ND} \times$ Age: $F\left(\mathrm{I}, 3^{6}\right)=0.24$, $p=0.63$, partial $\left.\eta^{2}=0.0 \mathrm{I}\right)$.

Phonological and lexical variables affected the children's ability to fastmap the words correctly and to make the correct link between the word form and the referent and, in a reciprocal way, affected the frequency of Unrelated Distracter and Foil errors. The PP and ND of the words, however, did not affect the number of Related Distracter errors. It would seem, therefore, that creating semantic links between items within a category is a robust process, that the creation of the network begins at the very outset of word learning, and is a process which is not readily affected by lexical and phonological variables.

\section{Post-hoc tests for potential confounds}

As the semantic categories and referent-non-word pairings were not counterbalanced across participants, post-hoc analyses were completed to identify whether specific semantic categories or specific non-word-referent pairings were significantly easier or harder for the children to learn, so, potentially, representing a confound.

Semantic categories. The influence of semantic category on the children's comprehension probe scores (choosing the Target) was analysed using a $2 \times 4$ within-subjects ANCOVA, the first factor being ND (high and low), the second factor being semantic category (toy, pet, food, vehicle) and with age as a covariate. There was no main effect of semantic category 
$\left(F(3, \mathrm{I} 08)=\mathrm{I} \cdot 63, p=0.19\right.$, partial $\left.\eta^{2}=0.04\right)$, no interaction between semantic category and ND $\left(F(3,108)=2 \cdot 27, p=0.18\right.$, partial $\left.\eta^{2}=0.04\right)$ and no interaction between semantic category and age $(F(3, \mathrm{I} \circ 8)=\mathrm{I} \cdot 66, p=0.09$, partial $\left.\eta^{2}=0.06\right)$. A similar $2 \times 4$ within-subjects ANCOVA with PP as the first factor (high and low), semantic category as the second factor (toy, pet, food, vehicle) and age as covariate also found no significant interaction between PP and semantic category $(F(3,108)=2 \cdot 08, p=0 \cdot 1 \mathrm{I}$, partial $\left.\eta^{2}=0.06\right)$. Therefore, semantic category does not appear to have been a confounding variable in these results.

Non-word-referent pairs. A homogeneity test was carried out on the standardized residuals of the items to test whether there was evidence for undue influence of any individual item(s). The result was not significant $\left(\chi^{2}(7)=8 \cdot 36, p=0 \cdot 30\right)$, suggesting that no individual item(s) had particular influence on the outcome.

\section{DISCUSSION}

The results of this study demonstrate that lexical and phonological variables exert separable influences on the fast-mapping abilities of children and that the influence of phonological variables (PP) change across development. This discussion explores the separate trajectories of influence of PP and ND, and potential explanations for these results. The results of this study are then placed in context with previous research findings, and future implications discussed.

\section{The developmental trajectory of fast-mapping biases}

Phonotactic Probability. The Phonotactic Probability of the novel nonwords exerted an influence on the children's fast-mapping abilities and this effect changed across the age range examined here $(3 ; \mathrm{OI}-5 ; 02)$, moving from a high PP advantage in the youngest children to a low PP advantage for the older children. This 'switch' in processing bias was not associated with increased efficiency in fast-mapping ability; indeed the children's ability to fast-map correctly remained constant across the trajectory. What, then, might be the driver of such a 'switch'?

For infants and young children a bias towards mapping more frequent sound combinations may arise from continuities from the properties of the earliest, infant speech processing mechanisms. That is, sensitivities to the statistical regularities of speech are thought to support the infant to abstract the phonetic regularities of the ambient language and so to segment words from the speech stream (Jusczyk, I997). The infant is thought to meet the challenge of identifying individual words from the continuous speech stream through the integration of a number of cues; prosodic, 
distributional, phonotactic and phonetic. Prosodic cues become available to infants aged approximately o;07 (Jusczyk, Houston \& Newsome, I999), but are not sufficient to segment all words successfully. By age o; 8, distributional cues are also used, so that the context within which a word is heard is used to support segmentation (Jusczyk, I 997). That is, the infant begins to 'remember' certain sound sequences, creating long-term representations of them. Highly frequent sound sequences (for example milk) create representations in the child's long-term store and then subsequent presentations of the sound sequence activate this representation and therefore are perceived as single units. Later the use of this word in a sentence helps the child to segment other sentence elements into individual words (e.g. chocolate milk would be segmented into two units due to the presence of the familiar sequence milk). It must be noted that this 'longterm representation' is not equivalent to word learning at this stage as it does not necessarily imply a link between the learned sound sequence and a semantic representation: rather it is thought that it constitutes a memory trace for the acoustic information of the word.

By age 0;09, phonotactic cues begin to be used, supporting the detection of highly probable and recurring sound sequences which would therefore most likely constitute a word, and also the identification of sound sequences with low probability (e.g. /db/ or $/ \mathrm{kt} /$ ), which would be most likely to constitute a word or syllable boundary (Mattys, Jusczyk, Luce \& Morgan, I 999), and by o; Io infants prefer to listen to words with high PP (Jusczyk, I 997). At a little over age o; I o, phonetic cues are integrated into the child's segmentation 'tool-kit' such that infants are sensitive to allophonic variation in speech sounds which are restricted to particular contexts (e.g. aspirated and unaspirated $/ \mathrm{t} /$ in word-initial and -final positions), to the distribution of these sounds within words, and are thus able to harness this knowledge to support them to detect word boundaries in continuous speech (Jusczyk, Hohne \& Bauman, I 999).

A bias in favour of learning sound sequences with higher phonotactic probabilities in the infant would seem, therefore, to be a highly adaptive strategy. First, with respect to distributional cues, learning high-frequency sequences would create a set of long-term representations for highly frequent sound combinations (e.g. is, mummy) which could then be used as distributional cues to further segment the speech stream and so identify more word units. A bias to do this for sequences which occur frequently would therefore support further segmentation more often than if lowfrequency sequences were learned. Second, with respect to phonotactic cues, identifying familiar sound sequences has been shown to support children's semantic mapping such that the presence of a familiar sound sequence is thought to instigate the 'search' for a probable referent. That is, the presence of sound sequences which the children recognize as 'word 
forms' has been shown to support both the individuation $(\mathrm{Xu}, 2002)$ and the categorization of objects (Waxman \& Lidz, 2006) in infants aged ०; ०9-I; ০০, and hence their semantic learning. Identifying and learning frequently occurring sound sequences, therefore, is an adaptive strategy for learning both word forms and word referents and so supports fast and efficient word learning.

Why then might children at the age of four 'switch' their word learning preference to a bias for words with low PP? It is possible that a strategy of learning word with high PP becomes inefficient at a certain critical mass of vocabulary. That is, as the lexicon grows, the need to learn words with a wider range of phonotactic patterns becomes more necessary and so a high PP bias would be maladaptive at this point. In addition, the older child, with a larger lexicon, is able to segment the speech stream into words using their existing lexical knowledge rather than their phonetic and phonotactic knowledge. That is, for children with a critical mass of lexical knowledge, when encountering a new word, on the majority of occasions it is only the novel word that is unknown to the child and so identifying word boundaries becomes a trivial task (e.g. Look at the big scary tyrannosaurus). For children with larger lexicons, directing attentional resources to learning high PP stimuli could, therefore, cease to provide a processing advantage for word learning.

Storkel and colleagues (2006) suggested that the low PP advantage they found in adult processing could be explained by assuming that PP was being used as a prelexical processing trigger for word learning such that novelty of the phonotactic pattern instigated new word learning. The results presented here corroborate their finding and in addition identify the time point at which this change in bias occurs in the developmental trajectory. We could therefore perhaps characterize infants and young children as 'statistical learners' who are primed to learn many new words at a very fast rate, and older children and adults as 'novelty learners' who learn words only when novelty has been identified.

What might be the driver of this change within the child's developing speech processing system? The crucial first step in creating a form-referent mapping is identifying that a novel word and/or referent has been encountered. If phonemic categories emerge over development at the sublexical/phonological level of processing, then older children will develop the ability to identify which fine-grained distinctions carry meaning, and therefore will become able to identify when word forms represent the presentation of a new word rather than an allophonic variation of a known word (e.g. $\operatorname{tog}$ versus $d o g$ ), even where a word has many neighbours. This is not the case for infants, and so, as described above, in order to identify a novel word, infants use a number of phonetic level cues (e.g. transitional probabilities, distributional properties of allophones, prosody), often integrating multiple cues simultaneously. As Werker and Curtin state: “one 
of the well known benefits of any higher order category is the increased processing efficiency it affords" (2005: 219). Hence, identifying novel words and word boundaries using phonemic representations and processing, rather than multiple and detailed phonetic cues, would create a processing advantage for the child, freeing up both the capacity and the functioning of the phonetic/indexical level of processing to support word learning in a different way. The sensitivity of the phonetic/indexical level of processing to $\mathrm{PP}$ could then be harnessed to trigger word learning in a different way: through the identification of novelty, as described by Storkel (2006). This change could perhaps be characterized as an increase in SALIENCY of low PP combinations for the child. Pierrehumbert (2003) suggests that the stimuli which are most salient for a child change over development as a result of changes in the nature of lexical knowledge and, in turn, the functioning of the speech processing system as a whole. The switch in PP bias reported here could represent empirical evidence in support of Pierrehumbert's claim.

The switch in PP bias, within a highly interactive system, could therefore be explained as emerging as a result of interactivity between levels of representation: changes in the influence of $\mathrm{PP}$ may reflect changes in the functioning of the phonetic/indexical level of processing which have emerged as a result of changes in the nature of representations at the sublexical/phonological level of processing.

In children, therefore, the PP effect on fast-mapping demonstrated here may not represent effects on processing which is LOCATED in the sublexical/ phonemic level of representation (as suggested in studies of adult processing; Vitevitch \& Luce, I 999). Rather, it may represent processing in the phonetic/indexical level of representation, whose function changes As A RESULT of change at the sublexical/phonemic level.

If changes at the sublexical/phonemic level of processing do indeed drive these changes in PP bias, then one would predict that the trajectory of influence of ND would reflect this change, such that words with low ND would be more easily learned than those with high ND, but that this advantage would decrease over development as phonemic categories emerge.

Neighbourhood Density. The Neighbourhood Density of the novel non-words exerted a separate and distinct influence on the children's fastmapping abilities as indicated by their abilities in the comprehension probe. That is, overall, low ND was advantageous to word learning. Inspection of the trajectory of influence of ND suggested that the disadvantage to fast-mapping exerted by high ND might have been reducing across development (see Figure 5). The interaction of ND and age was not statistically significant, with an effect approaching significance. Our results suggest that, if anything, the effects of neighbourhood density reduce with age, though one cannot be certain. 
The low ND bias suggests that children found it easiest to identify words with few neighbours as being novel and hence attempted to learn those words. However, they found this more difficult for words with many neighbours, and so, as they were less able to discriminate those words from words that already existed in their lexicons, they did not 'switch on' the word learning mechanism. As previously identified, one would predict that, as children become more able to make fine-grained distinctions between the words they hear and those in their lexicons, they should become able to trigger word learning even for words with many neighbours, and this change would be represented experimentally by a decreasing effect of ND on fast-mapping as children learn more words. Any observed changes in the trajectory of influence of ND on fast-mapping could, therefore, be conceptualized as tapping into changes at the sublexical/phonological level of processing, so that as phonemic categories emerge at the sublexical/ phonological level, children become able to distinguish between word forms more readily, thus increasing the specificity of their lexical processing.

The absence of a significant interaction between ND and age in the current study, however, means that we do not have robust support for this process of increasing specificity and emergence of phonemic categories in the age range studied. However, the findings from other studies may provide support. For example, a low ND advantage was also demonstrated for word learning in children aged $\mathrm{I} ; 4-2 ; 6$ by Storkel (2009). This study also found that the low ND advantage decreased with development. In addition, Storkel and colleagues (2006) found a high ND advantage for fast-mapping in adults. Given these previous findings, and the substantial trend towards reducing effects of ND with age in this study, it is possible that, had the current study had a larger sample size with a wider age range in the participants, the influence of ND on fast-mapping could be shown definitively to reduce over development, or, in fact, switch (from a low to a high ND advantage) at some point in the trajectory. It should be noted, however, that the findings with respect to adult fast-mapping (Storkel et al., 2006) were measured using an expression probe. It is therefore also possible that the influence of ND on fast-mapping may be constant across development (with a low ND advantage favouring fast-mapping) and that the observed differences between adults and children may occur as a result of differing task demands. Further work, with a wider age range of subjects, or exploring prospective longitudinal data, is needed to distinguish these possibilities.

\section{Placing the results in context}

Two issues emerge from the results of the present study which require further exploration if they are to be fully integrated with previous 
research findings; the nature of influence of $\mathrm{PP}$ on overall fast-mapping and the influence of PP on the creation of semantic networks and representations.

The influence of PP on fast-mapping. Previous fast-mapping studies in children carried out by Storkel and colleagues have consistently demonstrated a high PP advantage (Storkel, 200 I 2003; Storkel \& Rogers, 2000). The present study demonstrated a low PP advantage overall and a changing trajectory of influence beginning with a high PP advantage which changed to a low PP advantage across development.

The most obvious source of difference between the studies is the fact that Storkel and colleagues did not manipulate or control for the ND of the stimuli in the studies cited above. As PP and ND are highly correlated, Storkel's studies are best characterized as representing a comparison between high ND/PP words and low ND/PP words. However, the present study demonstrated an overall low ND and low PP advantage and so the influence of ND on Storkel's stimuli does not explain the high PP advantage in her results.

The crucial difference between Storkel's research and the current study is our focus on the DEVELOPMENTAL TRAJECTORY OF INFLUENCE of the variables. The present study examines the fast-mapping abilities of children aged $3 ; \mathrm{OI}-5 ; 02$, and Storkel considers children aged 3;02-6;04. Our results demonstrate a high $\mathrm{PP}$ advantage in the children aged between approximately 3;OI and 4;O0, and suggest that possibly a high ND advantage may emerge in the children aged 5;02 and above. Storkel's participants, therefore, may include a group for whom there is a high PP advantage $(3 ; 02-4 ; 00)$, a group with a low PP advantage $(4 ; 00-5 ; 02)$ and an older group with a high ND advantage $(5 ; 02-6 ; 04)$. On average, therefore, this group of children would be likely to show an overall high PP advantage. The current study, has demonstrated a more complex picture of the influence of PP on fast-mapping ability through the consideration of the additional factors of ND and of DEVELOPMENT.

The influence of PP on creating a semantic representation and network. In the current study, phonological and lexical variables affected the children's ability to fast-map novel words correctly. The PP and ND of the words, however, did not affect the number of related distracter errors made by the children. Choosing the Related Distracter at a level above chance would suggest the child had created a lexical representation which was sufficient for recognition but had either created a connection to the incorrect semantic representation (the other object in the semantic category), or to the semantic category, without being sure of the specific referent. The child had therefore made a link between the lexical representation and the SEMANTIC CATEGORY of the two novel referents but not to the specific semantic representation. It would seem, therefore, that creating semantic 
links between items within a category is a robust process for typically developing children and so was not readily affected by lexical and phonological variables. This result differs from previous research findings. Storkel (200I) found that PP did affect the proportion of Related Distracter errors made by children, such that a greater proportion of Related Distracter errors occurred for words with high PP than low PP.

Once again, a number of methodological differences between the studies may account for this disparity. Again, the most obvious candidate is that, in the present study, PP and ND are orthogonally varied. This could mean that the differences in phonotactic probabilities between the high and low PP conditions in the current study were less extreme than in Storkel's study and were not sufficiently different to exert an effect on processing. PP did exert an effect on all other response types and so this seems unlikely. Comparisons of the stimuli using a series of two-sample $t$-tests found no significant differences between the stimuli in the two studies except for the biphone probabilities of the low PP items $(t(6)=2.07 p=0.042$, one-tailed). In this case the stimuli used in the present study had higher biphone PP than those of Storkel (200I). This may explain the differences in results between the two studies.

There were, however, a number of additional methodological differences which should be considered. In order to minimize the possible confound of the influence of language knowledge on the word learning process across the trajectory, the story and sentence frames were simpler than those used by Storkel (200I), the target words always appeared in sentence final position, early developing semantic categories were used and the children heard ten rather than seven repetitions of each word.

Any of the above changes could therefore be the source of the difference between the studies. In fact ALL of the above changes TOGETHER could have resulted in a lower processing load for the current task than the task presented in Storkel (200I), and so improved overall success could have made this study less sensitive to the effects of PP. The children were performing well below ceiling, however, and so the processing load of the task was obviously still considerable for them. In addition, the numbers of Target, Unrelated Distracter and Foil responses were affected by PP. It would seem that recourse to processing load for an explanation of these differences in results might, therefore, be an oversimplification. An alternative source of the difference may be the choice of early developing semantic categories for the novel referents (toys, pets, food and vehicles in the current study, as opposed to toys, musical instruments, candy machines and pets in Storkel. 200I). Perhaps being able to integrate all of the novel words into an ExIsTING semantic category facilitated the creation of a semantic network for the novel words and this facilitative effect may have been sufficient to override that of PP. This explanation would be 
in keeping with Storkel's most recent study of CDI data (2009) where, for children over I; Io, words with many semantic neighbours were easier to learn than those with few. Therefore the semantic categories of 'vehicles' and 'food' may support greater integration of newly learned words into semantic networks than the categories 'candy machines' and 'musical instruments'.

\section{Limitations of the current study}

The study could have benefited from two improvements in methodology. First, a more balanced spread of participants across the age range studied would increase confidence that the results found were not overly influenced by one or two outliers. Inspection of the distribution of scores (Figures 3, 4 and 5) suggests that there are not any significant outliers in this data. However, future studies would be improved by tackling this issue.

A second improvement to the study would have been to randomize the referents across children and across semantic categories. This would have ensured that the potential confounds of semantic category and/or specific characteristics of the individual referents were removed. It should be noted, however, that post-hoc tests demonstrated that no such confounds were present for this data.

\section{Conclusion}

The developmental trajectory of word learning is a complex one involving separate, changing and interactive influences of $\mathrm{PP}$ and ND, and demonstrating changes in the nature of the speech processing mechanism in children across development. These differences suggest adaptive reorganization of the lexicon where changes in all levels of the processing system (semantic, phonetic/indexical, lexical and sublexical/phonological) emerge through interactive effects, which themselves change the nature of the word learning process. Interactivity in the moment of processing and across the developmental trajectory must be understood and the nature of change in the speech processing system as a whole described if fully specified developmental trajectories of speech processing and word learning are to be defined.

The current study provides empirical support for the need to consider developmental change in order to understand word learning in children, and for the need for prospective longitudinal data to explore the nature of change in speech processing and word learning across the developmental trajectory. 


\section{REFERENCES}

ASHA (1997). Guidelines for audiologic screening. Rockville, MD: ASHA.

Baayen, R. H., Piepenbrock, R. \& Gulikers, L. (I995). The CELEX Lexical Database (Release 2) (Publication, from Linguistic Data Consortium, University of Pennsylvania [Distributor]).

Beckman, M.E. \& Edwards, J. (2000). The ontogeny of phonological categories and the primacy of lexical learning in linguistic development. Child Development $7 \mathbf{I}(\mathbf{I})$, 240-49.

Dale, P. S. \& Fenson, L. (r996). Lexical development norms for young children. Behavioural Research Methods, Instruments and Computers 28, I 25-27.

De Cara, B. \& Goswami, U. (2002). Similarity relations among spoken words: The special status of rimes in English. Behavior Research Methods, Instruments, E Computers 34(3), 4I6-23.

De Cara, B. \& Goswami, U. (2003). Phonological neighbourhood density : Effects in a rhyme awareness task in five-year-old children. Fournal of Child Language 30(3), 695-7 Io.

Edwards, S., Fletcher, P., Garman, M., Hughes, A., Letts, C. \& Sinka, I. (I997). The Reynell Developmental Language Scales II: The University of Reading Edition. Windsor: NFER-Nelson.

Elliot, C. D., Smith, P. \& McCulloch, K. (1996). British Ability Scales Second Edition (BAS $I I)$. London: NFER-Nelson.

Fenson, L., Dale, P. S., Reznick, J. S., Thal, D. J., Bates, E., Hartung, J. P. et al. (I997). MacArthur Communicative Development Inventories: User's guide and technical manual. San Diego: Singular Publishing Group Inc.

Garlock, V. M., Walley, A. C. \& Metsala, J. L. (200 I). Age-of-acquisition, word frequency, and neighborhood density effects on spoken word recognition by children and adults. Fournal of Memory and Language 45(3), 468-92.

Golinkoff, R. M., Hirsch-Pasek, K., Bloom, L., Smith, L. B., Woodward, A. L., Akhtar, N. et al. (2000). Becoming a word learner: A debate on lexical acquisition. Oxford: Oxford University Press.

Grunwell, P. (1985). Phonological Assessment of Child Speech (PACS). Windsor: NFER-Nelson.

Hollich, G., Jusczyk, P. W. \& Luce, P. (2002). Lexical neighbourhood effects in I7-monthold word learning. Paper presented at the 26th Annual Boston University Conference on Language Development.

Jusczyk, P. W. (I997). Finding and remembering words: Some beginnings by English-learning infants. Current Directions in Psychological Science 6(6), I 70-74.

Jusczyk, P. W., Hohne, E. A. \& Bauman, A. (I999). Infants' sensitivity to allophonic cues for word segmentation. Perception \& Psychophysics 6r(8), I465-76.

Jusczyk, P. W., Houston, D. M. \& Newsome, M. (I999). The beginnings of word segmentation in English-learning infants. Cognitive Psychology 39, I 59-207.

Luce, P. A. \& Pisoni, D. B. (I 998). Recognizing spoken words: The neighborhood activation model. Ear and Hearing $\mathbf{1 9}(\mathrm{I}), \mathrm{I}-36$.

MacWhinney, B. (2000). The CHILDES project: Tools for analysing talk, 3rd edn, vol. 2 : The database. Mahwah, NJ : Lawrence Erlbaum Associates.

Maekawa, J. \& Storkel, H. L. (2006). Individual differences in the influence of phonological characteristics on expressive vocabulary development by young children. Fournal of Child Language 33(3), 439-59.

Mattys, S. L., Jusczyk, P. W., Luce, P. A. \& Morgan, J. L. (I 999). Phonotactic and prosodic effects on word segmentation in infants. Cognitive Psychology 38(4), 465-94.

McKean, C. (2009). Investigating the development of a developmental disorder: Mapping the trajectory of lexical development in Specific Language Impairment. Unpublished doctoral thesis, Newcastle University.

Morrison, C. M., Chappell, T. D. \& Ellis, A. W. (I 997). Age of acquisition norms for a large data set of object names and their relation to adult estimates and other variables. Quarterly Journal of Experimental Psychology 50A(3), 528-59. 
Pierrehumbert, J. B. (2003). Probabilistic phonology: Discrimination and robustness. In R. Bod, J. Hay \& S. Jannedy (eds), Probabilistic linguistics, 177-228. London: MIT Press.

Storkel, H. L. (200I). Learning new words: Phonotactic probability in language development. Fournal of Speech Language and Hearing Research 44(6), I 32 I-37.

Storkel, H. L. (2003). Learning new words II: Phonotactic probability in verb learning. Fournal of Speech Language and Hearing Research 46(6), I 3 I 2-23.

Storkel, H. L. (2004). Do children acquire dense neighborhoods? An investigation of similarity neighborhoods in lexical acquisition. Applied Psycholinguistics 25(2), 20I-22 I.

Storkel, H. L. (2009). Developmental differences in the effects of phonological, lexical and semantic variables on word learning by infants. Fournal of Child Language $\mathbf{3 6}$, 29I-32I.

Storkel, H. L., Armbruster, J. \& Hogan, T. P. (2006). Differentiating phonotactic probability and neighborhood density in adult word learning. Fournal of Speech, Language, \& Hearing Research 49, I I 75-92.

Storkel, H. L. \& Rogers, M. A. (2000). The effect of probabilistic phonotactics on lexical acquisition. Clinical Linguistics \& Phonetics 14(6), 407-425.

Swingley, D. \& Aslin, R. N. (2006). Lexical competition in young children's word learning. Cognitive Psychology 54, 99-1 32 .

Thomas, M. S. C., Annaz, D., Ansari, D., Scerif, G., Jarrold, C. \& Karmiloff-Smith, A. (2009). The use of developmental trajectories in studying developmental disorders. Fournal of Speech, Language, E Hearing Research 52(2), 336-88.

Vitevitch, M. S. \& Luce, P. A. (I 998). When words compete: Levels of processing in perception of spoken words. Psychological Science 9(4), 325-29.

Vitevitch, M. S. \& Luce, P. A. (I999). Probabilistic phonotactics and neighborhood activation in spoken word recognition. Fournal of Memory and Language 4o(3), 374-408.

Vitevitch, M. S. \& Luce, P. A. (2004). A Web-based interface to calculate phonotactic probability for words and non-words in English. Behavior Research Methods Instruments E Computers 36(3), $48 \mathrm{I}-87$.

Vitevitch, M. S. \& Luce, P. A. (2005). Increases in phonotactic probability facilitate spoken nonword repetition. Fournal of Memory and Language 52(2), I 93-204.

Vitevitch, M. S., Luce, P. A., Pisoni, D. B. \& Auer, E. T. (r999). Phonotactics, neighborhood activation, and lexical access for spoken words. Brain and Language. Special Issue: Mental lexicon 68( $\mathrm{I}-2)$, 306-3I I.

Walley, A. C., Metsala, J. L. \& Garlock, V. M. (2003). Spoken vocabulary growth: Its role in the development of phoneme awareness and early reading ability. Reading and Writing: An Interdisciplinary fournal I6(I), 5-20.

Waxman, S. R. \& Lidz, J. (2006). Early word learning. In W. Damon, D. Kuhn \& R. Siegler (eds), Handbook of child psychology, 6th edn, vol. 2: Cognition, perception and language, 299-335. Hoboken, NJ : Wiley.

Werker, J. F. \& Curtin, S. (2005). PRIMIR: A developmental framework of infant speech processing. Language Learning and Development I(2), 197-234.

Werker, J. F. \& Yeung, H. H. (2005). Infant speech perception bootstraps word learning. Trends in Cognitive Sciences 9(I 1 ), 5 I 9-27.

$\mathrm{Xu}, \mathrm{F} .(2002)$. The role of language in acquiring object kind concepts in infancy. Cognition $85,223-50$.

Ziegler, J. C. \& Goswami, U. (2005). Reading acquisition, developmental dyslexia, and skilled reading across languages: A psycholinguistic grain size theory. Psychological Bulletin $\mathbf{1 3} \mathbf{I}(\mathrm{I}), 3^{-29}$. 


\section{APPENDIX}

Page Script

I Jim and Bob are Aliens. They live on the planet Plop. There are lots of strange things on the planet Plop. Shall we go and see?

2 Jim and Bob are going shopping. What will they buy today?

3 First they visit the toy shop.

4 Jim says I want a /teIn/. Bob says I want a /horf/. Here is the /teIn/. Here is the /horf/.

5 Look! Jim has the /teIn/. Look! Bob has the /horf/. They are very happy with their new toys.

[Comprehension probe]

5 This one is the /teIn/. Jim bought the/teIn/. This one is the /horf/. Bob bought the /horf/. Which toy do you like best? That one is the ...... (name). I like the ...... (name other toy).

6 Now they visit the pet shop. What will they buy today?

7 Jim says I want a /baIn /. Bob says I want a /joj/. Here is the /baIn/. Here is the /joj/.

8 Look Jim has the /bain/. Look Bob has the /jof/. They love their new pets.

[Comprehension probe]

8 This one is the /baIn/. Jim bought the /baIn/. This one is the /jof/. Bob bought the /jof/. Which pet do you like best? That one is the ...... (name). I like the ...... (name other pet).

9 Now they are feeling hungry. Time to go to the café

Io Jim says I want a /gek/. Bob says I want a /heIm/. Here is the /gek/. Here is the /heIm/.

I I Look Jim is eating the /gek/. Look Bob is eating the /heIm/. They are enjoying their yummy food.

[Comprehension probe]

I I This one is the /gek/. Jim bought the /gek/. This one is the /heIm/. Bob bought the /herm/. Which food do you like best? That one is the ...... (name). I like the ...... (name other food).

I2 Now they are getting tired it's time to go home. They go to the rocket stop to catch a rocket.

I3 Jim says I want to catch a /han/. Bob says I want to catch a / eeIt/. Here is the /han/. Here is the $/$ eit/.

I4 Look Jim is in the /han/. Look Bob is in the / Jert/. Whoosh away they fly on their rockets.

[Comprehension probe]

I 4 This one is the /han/. Jim caught the /han/. This one is the / Sert/. Bob caught the / $\mathrm{fert} /$. Which space ship do you like best? That one is the ...... (name). I like the ...... (name other spaceship).

I 5 Now it's time for bed and Jim and Bob are remembering all the things they have done today. They visited a toy shop. They bought a /teIn/ ...... and a /horf/. Here is the /teIn/ ...... and here is the /horf/. They visited the pet shop. They bought a /bain/..... and a $/ \mathrm{j} 0 \mathrm{~J} /$. Here is the $/ \mathrm{baIn} / \ldots \ldots$ and here is the $/ \mathrm{j} \mathrm{j} \mathrm{J} /$. They visited a café. They ate a /gek/ ...... and a /heIm/. Here is the /gek/...... and here is the /heIm/. Then they caught a rocket home. They rode on a $/ \mathrm{han} / \ldots \ldots$ and a $/$ Sert $/$. Here is the $/ \mathrm{han} / \ldots \ldots$ and here is the / Sert/. Goodnight Jim and Bob

[Comprehension probe] 
Can you find the toys Jim and Bob bought in the toy shop? That's right you've found the /teIn/...... and you've found the /horf/. Jim bought a /teIn/. Bob bought a /horf/.

Can you find the pets Jim and Bob bought in the pet shop? That's right you've found the /bain/...... and you've found the /jof/. Jim bought a /baIn /. Bob bought a /jof/.

Can you find the food Jim and Bob bought at the café? 'That's right you've found the /gek/ ...... and you've found the /heIm/. Jim bought a /gek/. Bob bought a /heIm/.

Can you find the rockets Jim and Bob rode home in? That's right you've found the /han/ $\ldots . .$. and you've found the $/ \int$ eIt/. Jim rode in the $/ \mathrm{han} /$. Bob rode in the $/ \int$ eIt/.

[Comprehension Probe]

TAB LE 3. Number of correct responses on comprehension probe (Target) across assessment points

\begin{tabular}{lccc}
\hline & Assessment I & Assessment 2 & Assessment 3 \\
\hline$M$ & $4 \cdot 05$ & $4 \cdot \mathrm{I} 6$ & $4 \cdot \mathrm{I} 8$ \\
$S D$ & $(\mathrm{I} \cdot 92)$ & $(\mathrm{I} \cdot 88)$ & $(\mathrm{I} \cdot 69)$ \\
\hline
\end{tabular}

NOTE: maximum score $=8$. 